\title{
Gamma radiation as a source of information about the characteristics of celestial bodies
}

\author{
Marek Przyborski ${ }^{1}$, Zuzanna Siemieniago ${ }^{1,{ }^{*}}$ \\ ${ }^{1}$ Gdańsk University of Technology, Faculty of Civil and Environmental Engineering, Gdansk, Poland
}

\begin{abstract}
The Universe is a violent and hostile environmental. Remote sensing methods give the possibility to search for the answer to the most fundamental question that humankind has ask, how the universe works? In this paper we would like to take a closer look at the some specific objects in the Universe that their influence on its evolution is still a matter of research all over the world. The Gamma Bursts are one of the most dangerous phenomenon that we have ever discovered. Astronomers may observe them also when watching and searching for another form of energy propagated through the time and space gravitational waves caused by binary systems of neutron stars. In the following we would like to explain the fundamentals of multi-messenger astronomy.
\end{abstract}

\section{Introduction}

Gamma ray bursts accompany one of the most dramatic events that occur in the universe. The observation of gamma radiation allows the discovery of further celestial bodies and the study of already known ones like pulsars, black holes, nuclei of active galaxies or dark matter [12, $16,21,3]$. In space, there are constantly many processes emitting huge amounts of energy, such as star bursts or asteroid collisions that cause weak gamma-ray bursts [12]. Electromagnetic waves accompanying events occurring in the Universe arise in microscopic processes, strongly interact with the surrounding matter, are absorbed and dispersed. The information they provide relates to the moment of the last contact with matter, e.g. the surface of the last scattering, which may be the surface of the star [3].

Gamma bursts can be divided, in terms of duration, into short and long [21]. Long, lasting from several seconds to even several minutes, called hibernate, accompanied by powerful supernova explosions [14]. Short in turn, they last only a fraction of a second and result from the merging of neutron stars-objects created after supernova explosions [17].

Neutron stars are objects with small diameters, not exceeding $25 \mathrm{~km}$, but they have very high density of matter. Bearing in mind the principle of conservation of the angular momentum and the characteristics of the object, it is not surprising that its incredible speed of rotation, which reaches even more than 700 revolutions per second. They were created as a result of the collapse of the nucleus of a star with a large mass (8-10 solar masses). Such a phenomenon brings about a rapid increase in the magnetic field, hence neutron stars have a magnetic field that is at least one hundred million times more powerful than the Earth's magnetic field [12]. Such a magnetized, fast-rotating star, hereinafter referred to as a pulsar, generates electricity, which is associated with the emission of electromagnetic radiation [13]. This is most often radio or X-ray radiation.

The discovery of pulsars in the 1960s gave rise to their further observations and research. Soon a pulsar was identified, called the Hulse-Taylor pulsar, with a variable period, from the names of the discoverers. It turned out to be part of the double star system. The general theory of relativity shows that accelerating masses should produce gravitational waves carrying quanta of energy, so that stars circling in orbits lose energy and the diameters of orbits decrease. This results in the stars' constant approaching each other and finally their collision. The final effect of such a collision will be the creation of a black hole. The material released as a result of the collision is ejected into space at a considerable speed. It reaches the border of the dual system, where it meets interstellar matter. Then, shock waves create electromagnetic radiation [5].

Currently, the effect of the above events is also observed as gamma radiation. Unfortunately, the flare itself does not allow for precise positioning of its source and distance. Also the very nature of gamma radiation makes it difficult to find the location of the source. The emission of radiation flux looks different depending on the case. During long gamma-ray bursts, the radiation is emitted along the axis of rotation of the collapsing star. The phenomenon takes place differently in the situation of the collision of neutron stars, then the emission occurs in the direction perpendicular to the orbital plane of the connecting objects. Looking along the beams we will see a strong flare, but if the detector is directed at a different angle, it may not register the signal at all.

Very often, the accompanying phenomenon is the presence of X-rays, which have only a little less energy

\footnotetext{
* Corresponding author: z.siemieniago@gmail.com
} 
than gamma, the emission of which is longer, and allows a more accurate determination of the location of the source of the flash [21]. The glow of $\mathrm{X}$ - ray and visible light seen on Earth connected with the flash is visible even above one day, which in comparison with the duration of a short gamma ray gives a better chance of observing the phenomenon.

Gamma radiation is not directly recorded from Earth [1]. The atmosphere that absorbs some ranges of wavelengths, e.g. lethal to cells of living organisms gamma rays.

There are indirect methods, e.g. Cherenkov radiation registration, which is emitted as a result of the interaction of gamma photons with the Earth's atmosphere. Said radiation is emitted when the charged particle moves at a speed greater than the phase velocity of light in the given medium, in a precisely defined direction at an acute angle to the direction of motion of the particle [2].

The technology described is used by projects such as CTA (Cherenkov Telescope Array) or VERITAS (Very Energetic Radiation Imaging Telescope Array System) [17]. Both are equipped with terrestrial telescopes with a mirror of 4 to 24 meters. In these, and similar projects, sensitive charged-coupled device (CCD) cameras or multi-pixel photomultiplier tubes (abbreviated as PMT) are used for registration.

Observations should meet several conditions to identify the gamma-ray burst:

- have the same intensity on each of the telescopes,

- appear in the same place in each camera,

- be a point phenomenon.

Such assumptions allow to exclude the possibility of observing the background radiation, not the gamma ray [14].

It should be emphasised that Cherenkov radiation is scattered relative to the gamma ray beam. It is usually registered as a several-kilometre area. The centroid of this field should represent the source of the emitted wave [4].

A lot of information is provided by the international space gamma ray observatory (Gamma-ray Large Area Space Telescope) today called the Fermi Gamma-ray Space Telescope from the name of the professor, pioneer in high energy physics, Enrico Fermi. Launched in 2008, the Fermi telescope carries out continuous observations of the sky in the field of high energy waves. It is equipped with two instruments:

- LAT - Large Area Telescope - detector with high sensitivity and resolution, covers about $1 / 5$ of the whole sky,

- GBM - Gamma-ray Burst Monitor - a supplementary device for the LAT, has a very short response time, which allows the registration of gamma-ray bursts [20].

The work of both instruments differs from each other. When the gamma ray gets to the LAT, it affects one of the 16 tungsten sheets. This interaction transforms the gamma ray into electron and positron, which allow you to follow the movement. Thanks to such transformation, LAT is able to determine the direction of radiation. GBM detectors capture X-rays or low-energy gamma rays. At the moment when the rays fall on one of the 12 detectors made of sodium iodide, a flare of the registered photomultiplier occurs. By comparing the signal speed of at least $4 \mathrm{GBM}$ detectors it is able to triangulate to determine the position of the source of the burst.

The combination of two instruments has allowed many discoveries, including pulsars, identification of the largest gamma-ray bursts, background radiation sources [13]. A significant part of the data from both the LAT and GBM observations is available on the Internet on the website: https://fermi.gsfc.nasa.gov/.

\section{Collisions of neutron stars}

The collisions of neutron stars are phenomena which, according to Einstein's theory, should be accompanied by the appearance of gravitational waves. The radiation generated during this explosion, in addition to radio ranges, should also be supplemented with the visible range. The observation of this event in the visible range and observations in the field of gamma and X-ray radiation are a kind of warning signal, which proves that we can also deal with the emission of gravitational wave [5]. Thanks to observations conducted in various ranges, we are able to identify the source of the gravitational wave. In this way a new type of astronomy was created which was initiated with the creation of the Laser Interferometer Gravitational-Wave Observatory (LIGO) project [10]. One of the last events recorded by LIGO observatories probably occurred at a distance of 130 million light years from Earth, in the galaxy NGC 4993. The distance indicates that astronomers have to deal with the closest, ever observed, source of gravitational waves that have been recorded [11]. It is also one of the closest registered gamma-ray bursts. According to the researchers, newly detected phenomena is also signalling the presence of socalled kilonova - an object whose existence theorists have been predicted for over 30 years. Kilonova can be the main objects responsible for the proliferation of very heavy particles in space, including gold and platinum [6]. According to the researchers, simultaneous detection of gravitational waves and their electromagnetic counterpart (including visible light) from the same source marks the beginning of a new era in astronomy, which they describe as "multi-messenger astronomy" $[11,18]$. The signal of gravitational waves (event marked GW170817 its localization is presented in Figure 1) was registered on 17 August 2017 at 14:41 CEST is a twin detector LIGO, located in Hanford, Washington, and Livingston, Louisiana (USA) [11]. 


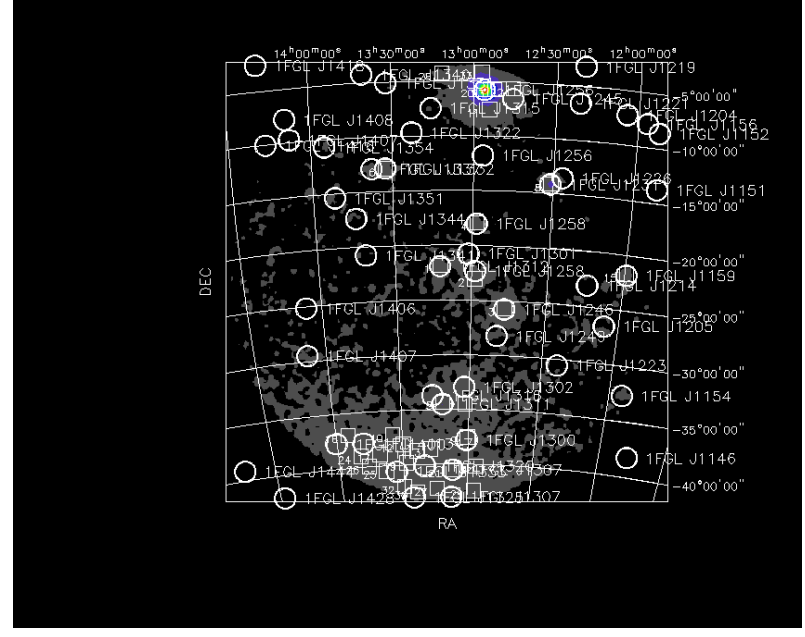

Fig. 1. Location of GW170817 (source: http://cds.u-strasbg.fr).

The participation of the Virgo Observatory allowed for the specification of the sky area from which the signal came. Then, the work involved observatories designed to study electromagnetic waves in various wavelength ranges, combing the sky in search of an electromagnetic equivalent for the source of gravitational waves $[15,19]$. Characteristic of the so-called gravitational waves, in this case, the tweet lasted almost two minutes and the last 30 seconds are shown in the spectrogram below Fig. 2 .

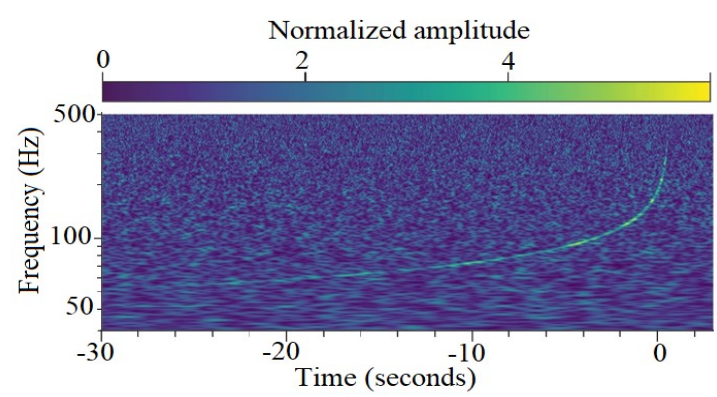

Fig. 2. Spectrogram of signal CHIRP (source: www.ligo.org/).

\section{Spectrogram}

Detailed analysis of this signal indicates that the observed system is much less massive, and the mass of the system components correspond to the double system of neutron stars [18]. The possibility of observing the evolution of such a system in the range other than the spectral ranges used so far, gives the possibility of a thorough analysis of one of the most extreme objects found in the Universe. The fact that these objects are created during the explosion of supernova stars, where the matter collapses to a density many times greater than the density of atomic nuclei and we are not able to reproduce this process on Earth, causes that the data collected during the observation of the evolution of such a dual system is very unique [17]. Registered by the Fermi satellite, simultaneous with the collapse, gamma-ray burst, was named GRB 170817A [7]. The characteristic curve of the radiance changes from this source agrees with theoretical models of the Kilonova, or clouds of neutron-sized matter remaining after the collision of neutron stars, which shines due to radioactive decays of elements. This event made it possible to test the correctness of the new method of measuring distances in the Universe, independent of the previously used socalled methods of "standard candles". The observed double system can be seen as a "standard siren" [18] providing a distance that it compares with the known escape velocity of the NGC 4993 galaxy, $\vartheta_{H}$, i.e. using Hubble's law:

$$
\vartheta_{H}=H_{0} d
$$

where :

$\vartheta_{H^{-}}$recessional velocity, the velocity of galaxy getting away, expressed in $\mathrm{km} / \mathrm{s}$;

$H_{0}$ - the constant of proportionality, Hubble constant expressed in $\mathrm{km} / \mathrm{s} / \mathrm{Mpc}$ :

$$
H_{0}=70_{-8}^{+12} \mathrm{~km} \cdot \mathrm{s}^{-1} M p \mathrm{c}^{-1}
$$

$d$ - distance to the galaxy masured in Mpc.

Thus, in this way, we have also independent from other methods of measurement of the Hubble constant, and thus a new estimation of the rate of Universe spread, which is compatible with measurements from supernovae and microwave background radiation measurements. Thanks to the measurements made during the event GW 170817, a comparison of the time shift between gravitational waves and photons was also made, which allowed to estimate the speed of gravitational waves, which turns out to be almost identical to the speed of light.

During the observation of the GW 170817 event, scientists launched one of the largest ever target observation campaigns, seeking the equivalent of a source of gravitational waves in many ranges of electromagnetic wavelength. As part of this action, one of the first, the optical equivalent of the source of gravitational waves was noticed by the infrared VISTA (Visible \& Infrared Survey Telescope for Astronomy) telescope. A number of other European Southern Observatory (hereinafter ESO) telescopes were used, such as the Very Large Telescope (VLT), the VLT Survey Telescope (VST), the New Technology Telescope (NTT), as well as the Atacama Large Millimeter Array (ALMA) radio telescope network and other partner instruments working in the ESO observatories in Chile. These instruments followed the object for subsequent days and weeks after detection. The observations were also conducted from space, e.g. by the Hubble Space Telescope. The NGC 4993 galaxy, where neutron stars joined, was observed by the OGLE team led by prof. Andrzej Udalski. The Polish project Pi of the Sky, implemented by the Faculty of Physics of the University of Warsaw, the National Centre for Nuclear Research of the Polish Academy of Sciences and the Centre of Theoretical Physics of the Polish Academy of Sciences, whose main goal is to search for optical flashes accompanying gamma flares. 


\section{Big Bang and LIGO}

Thanks to the LIGO detectors, we have also been able to study the earliest moments of the Universe. The basis for these analyses are earlier discoveries of gravitational waves, the source of which was the collision of black holes. The gravitational waves discovered in the Advanced LIGO experiment, created by the double system of black holes, give one more opportunity to learn what the beginning of the universe looked like and allow to reach even further into the past. As assumed in the modern version of the Big Bang theory, at the very beginning the Universe underwent a phase of rapid expansion, so-called inflation, during which the original gravitational waves were generated. Like the relic of neutrinos and electromagnetic radiation, they fill the Universe today [10]. Unfortunately, the amplitude of the primary waves with frequencies measured in the Advanced LIGO experiment is much smaller than the sensitivity of this instrument. The waves of sizes comparable to the size of the observable universe have much greater amplitude, but to detect them, we would need such a detector size. The primary gravitational waves, however, have an effect on the polarity of the background radiation, so the radiation can act as a specific detector. The background radiation is partially polarized as a result of Thomson's dispersion on free charged particles, primarily electrons. However, for the scattered photons to be polarized, the electron radiation falling on the electron must have such a distribution that the directions corresponding to the maximum and minimum radiation intensity are set at right angles to each other. The gravitational waves passing through the ionized medium allow to generate such a distribution.

The directions of radiation polarization projected onto the observer's blue sphere will create a zero-source field. By analogy with the magnetic field in electromagnetism this type of polarization is called the B mode, in contrast to the $\mathrm{E}$ modes generated primarily by ordinary scalar disturbances. Detection of $\mathrm{B}$ mode polarization of relict radiation would be indirect evidence of the existence of primary gravitational waves and confirmation of the epoch of cosmological inflation in the early Universe. Thanks to that, we could find out what the Universe looked like only about a $10^{-36}$ second after the initial singularity $[8,9]$.

The detection of modes B polarization of background radiation is one of the most important goals of present and planned experiments on relict radiation. In 2014, such a detection was reported by the BICEP 2 experiment team $[8,9]$. However, thanks to the data from the Planck satellite, it quickly turned out that the observed B mods are related to the polarization of radiation emitted by our Galaxy. So the search for traces of the original gravitational waves is still going on. However, the lack of detection already allows to estimate the upper limit of energy at which inflation could occur. This boundary is about $10^{16} \mathrm{GeV}$ and so, there are several orders of magnitude lower than the Planck energy, at which gravity separated from the other interactions.

The answer to the question about even earlier moments of the Universe depends on what happened before the inflation era. It can be assumed that with the already mentioned Planck energy some physical processes have occurred, the traces of which can be preserved in the properties of relict radiation, neutrino background or primary gravitational waves. However, to better understand this, we need a quantum theory of gravity describing the interaction with the Planck energy. It is worth mentioning that according to some theories aspiring to the quantum theory of gravity in the era of such a high energy density loses the meaning of the notion of time known to us, and the smallest unit of time, having a physical meaning, is about a $10^{-44}$ second.

\section{Summary}

The events presented in this paper, followed, among others, by LIGO observatories, are an example of research in the new era in astronomy. Multi-messenger astronomy gives us a wide range of research possibilities of the Universe. Until now, astronomy was associated with longlasting phenomena, because such observations could be observed in the spectral ranges. Observations of gravitational waves give the possibility of recording phenomena lasting very shortly, in the order of seconds. The widespread availability of these data gives researchers the opportunity to improve their methods, improve software codes, and understand the phenomenon that Albert Einstein predicted 100 years ago.

\section{References}

1. W. Romanishin, An Introduction to Astronomical Photometry Using CCDs. Image (Rochester, N.Y.), 167. (2002)

2. J. B. Kepka, IV.5. Promieniowanie Czerenkowa, 2-6 (1984) (in Polish)

3. A. Subramaniam, R. Sagar, Multicolor CCD Photometry and Stellar Evolutionary Analysis of NGC 1907, NGC 1912, NGC 2383, NGC 2384, and NGC 6709 Using Synthetic Color-Magnitude Diagrams. The Astronomical Journal, 117, 2, 937961 pp. (1999) DOI:10.1086/300716

4. J. Aleksić, J. Ric, M. Martínez, Optimized analysis method for indirect dark matter searches with Imaging Air Cherenkov Telescopes. Proceedings of Science. (2013) DOI:10.1088/14757516/2012/10/032

5. J. Centrella, Gravitational wave astrophysics: Opening the new frontier. AIP Conference Proceedings, 1381, pp. 98-116 (2011) DOI: $10.1063 / 1.3635827$

6. B.P. Abbott, R. Abbott, T.D. Abbott, F. Acernese, K. Ackley, C. Adams, et al., A gravitational-wave standard siren measurement of the Hubble constant. Nature, 551, 7678, 85-98 pp. (2017) DOI : $10.1038 /$ nature24471

7. S.J. Smartt, T.W. Chen, A. Jerkstrand, M. Coughlin, E. Kankare, S.A. Sim, et al., A kilonova as the electromagnetic counterpart to a gravitational-wave 
source. Nature, 551, 7678, 75-79 pp. (2017) DOI :10.1038/nature24303

8. A. Goldstein, P. Veres, E. Burns, M.S. Briggs, R. Hamburg, D. Kocevski, et al., An Ordinary Short Gamma-Ray Burst with Extraordinary Implications: Fermi-GBM Detection of GRB 170817A. (2017) DOI:10.3847/2041-8213/aa8f41

9. P.A.R. Ade, R.W. Aikin, D. Barkats, S.J. Benton, C.A. Bischoff, J.J. Bock, et al., Detection of B -mode polarization at degree angular scales by BICEP 2 . Physical Review Letters, 112, 24 (2014) DOI:10.1103/PhysRevLett.112.241101

10. P.A.R. Ade, N. Aghanim, Z. Ahmed, R.W. Aikin, K.D. Alexander, M. Arnaud, et al., Joint Analysis of BICEP2/Keck Array and Planck Data. Physical Review Letters, 114, 10, 1-17 pp. (2015) DOI:10.1103/PhysRevLett.114.101301

11. S. S. Kimura, K. Murase, I. Bartos, K. Ioka, et al., Trans-Ejecta High-Energy Neutrino Emission from Binary Neutron Star Mergers (2018) arxiv.org/abs/1805.11613v1

12. S. Kaufmann, O. Tibolla, Ancient Pulsar Wind Nebulae as a natural explanation for unidentified gamma-ray sources. (2018) arxiv.org/abs/1805.11522v1

13. R. T. Bartels, T. D. P. Edwards, C. Weniger, Bayesian Model Comparison and Analysis of the Galactic Disk Population of Gamma-Ray Millisecond Pulsars. (2018) arxiv.org/abs/1805.11097

14. Y. Mizumura, A. Takada, T. Tanimori, Systematic Study of Nuclear Gamma-Ray Spectra of One Hundred Super Novae Expected by Future Nuclear Gamma-Ray Imaging Spectroscopic Observations. (2018) arxiv.org/abs/1805.07939

15. V. Tatischeff, A. De Angelis, M. Tavani, I. Grenier, et al., The e-ASTROGAM gamma-ray space observatory for the multimessenger astronomy of the 2030s. (2018) arxiv.org/abs/1805.06435

16. H.E.S.S. Collaboration, H. Abdallah, A. Abramowski, F. Aharonian et al., Search for $\gamma$-ray line signals from dark matter annihilations in the inner Galactic halo from ten years of observations with H.E.S.S. (2018) arxiv.org/abs/1805.05741

17. S. Celli, G. Morlino, S. Gabici, F. Aharonian, Supernova remnants in clumpy media: particle propagation and gamma-ray emission. (2018) arxiv.org/abs/1804.10579

18. J. Wei, X. Wu, H. Gao, Cosmology with Gravitational Wave/Fast Radio Burst Associations. (2018) arxiv.org/abs/1805.12265

19. The LIGO Scientific Collaboration, the Virgo Collaboration: B. P. Abbott, R. Abbott, T. D. Abbott, et al., GW170817: Measurements of neutron star radii and equation of state. (2018) arxiv.org/abs/1805.11581

20. G. Wang, Z. Wang, Searching for $\gamma$-ray Counterparts to Very Faint X-Ray Transient Neutron Star binaries. (2018) arxiv.org/abs/1804.09871
21. V. Liccardo, M. Malheiro, M. S. Hussein, T. Frederico, Nuclear processes in Astrophysics: Recent progress. (2018) arxiv.org/abs/1805.10183 